\title{
Development and validation of comprehensive closed formulas for atmospheric delay and altimetry correction in ground-based GNSS-R
}

\author{
T. Nikolaidou, Student Member, IEEE, M. C. Santos, S. D. P. Williams, and F. Geremia-Nievinski, \\ Member, IEEE
}

\begin{abstract}
Radio waves used in Global Navigation Satellite System Reflectometry (GNSS-R) are subject to atmospheric refraction, even for ground-based tracking stations in applications such as coastal sea-level altimetry. Although atmospheric delays are best investigated via ray-tracing, its modification for reflections is not trivial. We have developed closed-form expressions for atmospheric refraction in ground-based GNSS-R and validated them against raytracing. We provide specific expressions for the linear and angular components of the atmospheric interferometric delay and corresponding altimetry correction, parameterized in terms of refractivity and bending angle. Assessment results showed excellent agreement for the angular component and good for the linear one. About half of the delay was found to originate above the receiving antenna at low satellite elevation angles. We define the interferometric slant factor used to map interferometric zenithal delays to individual satellites. We also provide an equivalent correction for the effective satellite elevation angle such that the refraction effect is nullified. Lastly, we present the limiting conditions for negligible atmospheric altimetry correction (sub-cm), over domain of satellite elevation angle and reflector height. For example, for 5meter reflector height, observations below $20^{\circ}$ elevation angle have more than 1-centimeter atmospheric altimetry error.
\end{abstract}

Index Terms-GPS, GNSS, GNSS-R, reflectometry, atmospheric delay, tropospheric delay, ray-tracing, radio wave propagation.

\section{INTRODUCTION}

G LOBAL Navigation Satellite System Reflectometry (GNSS-R) has been widely applied for coastal sea level altimetry from ground-based tracking stations [1], [2], [3]. Unfortunately, GNSS-R altimetry suffers from atmospheric refraction [4]. Its linear and angular components induce, respectively, speed retardation and direction bending on both the direct and reflected rays. The consequence is an interferometric propagation delay, between the two ray paths, as compared to the idealization of propagation in vacuum. The atmospheric delay yields an atmospheric altimetry bias defined by the altimetry retrieval method employed. For example, for a $10-\mathrm{m}$ reflector height, the bias exceeds $0.5 \mathrm{~m}$ for a satellite near

\footnotetext{
Manuscript received ___ ; revised __; accepted __. TN acknowledges funding from Mitacs. FGN acknowledges funding from CNPq (457530/20146, 433099/2018-6) and Fapergs (26228.414.42497.26062017).

T. Nikolaidou and M. C. Santos are with Department of Geodesy and Geomatics Engineering, University of New Brunswick, Fredericton, NB, Canada (e-mail: thalia.nikolaidou@unb.ca, marcelo.santos@unb.ca).
}

the horizon [5] and is thus significant even for near-surface configurations [6]. It increases exponentially with satellite elevation angle and linearly with reflector height, i.e., distance between the antenna and the surface.

Frequently in the literature, the atmospheric delay in GNSS$\mathrm{R}$ is simplified as "twice the delay experienced between the specular point and the reception antenna" [6], [9]. This is based on the assumption that the effect of the atmosphere above the receiver cancels out, and only those effects due to the bottom layer, between the surface and the receiver, affect the altimetric range [10], [11]. However, atmospheric models developed for direct or line-of-sight propagation, as used in GNSS positioning, cannot compensate for the total atmospheric refraction effect [12]. That is because angular refraction, experienced by the incoming rays in the portion of the atmosphere above the antenna, does not necessarily cancel out when forming the interferometric atmospheric delay.

The most accurate modeling for the atmospheric delay in GNSS-R is provided by the raytracing technique [7], [13] . In its rigorous formulation, it requires solving a direct two-point boundary value problem (BVP), involving transmitting satellite and receiving antenna, followed by a three-point BVP, involving additionally the reflecting surface [2][5]. We have found that rigorous raytracing may be simplified with rectilinear raytracing if each direct and reflected ray paths are replaced by the respective refracted or apparent tangent directions [12]. A detailed description of an interferometric raytracing procedure is given in [5].

Although raytracing results are general and comprehensive, they require software that is not always available for GNSS-R researchers. Furthermore, most atmospheric raytracing software used in space geodesy are designed for direct only ray paths. Here, we develop and validate closed-form expressions for the atmospheric delay and corresponding altimetry correction for use in ground-based GNSS-R. They are parameterized in terms of ancillary atmospheric information (average layer refractivity and elevation bending angle) besides the independent geometrical variables (reflector height and satellite elevation angle). Refractivity and bending may be modeled as functions over space (latitude, longitude, altitude)

S. D. P. Williams is with National Oceanography Centre, Liverpool, UK, (email: sdwil@noc.ac.uk).

F. Geremia-Nievinski is with Department of Geodesy, Institute of Geosciences, Federal University of Rio Grande do Sul, Porto Alegre, Brazil (email: felipe.nievinski@ufrgs.br). 
and time (year, day of year, time of day). We validate the derived closed-form expressions by comparison to raytracing results, both rigorous and rectilinear.

Besides the main results above, we also provide closed formulas for derived quantities, such as the interferometric slant factor, used to map interferometric zenithal delays to individual satellites, and an elevation angle correction, such that the full atmospheric refraction effect is nullified. Finally, we provide the limiting conditions for significant atmospheric altimetry correction, in terms of satellite elevation angle and reflector height; in other words, we indicate the observation conditions under which linear and angular atmospheric refraction is negligible in ground-based GNSS-R.

\section{BACKGROUND}

For the sake of keeping this work more self-contained, in this Background section we briefly recapitulate the essential concepts of atmospheric refraction in GNSS-R; for details, the reader is referred to [5].

\section{A. Atmospheric Delay Formulation}

The atmospheric delay $d=L-D$, is defined in terms of two intrinsic radio propagation quantities: the vacuum distance, $D=\left\|\boldsymbol{r}_{1}-\boldsymbol{r}_{2}\right\|$ and the radio length, $L=\int_{\boldsymbol{r}_{1}}^{\boldsymbol{r}_{2}} n d l$, where $n$ is the index of refraction. The vector norm is evaluated given any two points, such as transmitting satellite and receiving antenna, involved in the direct vacuum distance, $D_{d}=\left\|\boldsymbol{r}_{\mathrm{sat}}-\boldsymbol{r}_{\mathrm{ant}}\right\|$. The integral is evaluated along the bent ray path, of infinitesimal arc length $d l[5]$.

Introducing further the curve range, $R=\int_{r_{1}}^{r_{2}} 1 d l$, allows the definition of two classical atmospheric delay components,

$$
d=(L-R)+(R-D)=d^{a}+d^{g}
$$

The first quantity on the right-hand side of eq.(1), $d^{a}$, is the along-path atmospheric delay:

$$
d^{a}=L-R=\int_{r_{1}}^{r_{2}} N d l
$$

where $N=n-1$ is the refractivity. The second quantity, $d^{g}$, in the end of eq.(1), is the geometric atmospheric delay:

$$
d^{g}=R-D
$$

The definitions above (eq.1-3) can be applied to either the direct path, yielding $d_{d}=L_{d}-D_{d}=d_{d}^{a}+d_{d}^{g}$, or to the reflection path, , yielding $d_{r}=L_{r}-D_{r}=d_{r}^{a}+d_{r}^{g}$. The latter involves the refracted specular point on the surface $\boldsymbol{r}_{\mathrm{sfc}}^{\prime}$ (not to be confused with the vacuum specular point, $\boldsymbol{r}_{\mathrm{sfc}}$ ) [5].

Corresponding interferometric quantities result from the difference between reflection and direct quantities, for example: interferometric vacuum distance, $D_{i}=D_{r}-D_{d}$; interferometric radio length, $L_{i}=L_{r}-L_{d}$; and interferometric curve range: $R_{i}=R_{r}-R_{d}$. Hence, the interferometric atmospheric delay follows from two equivalent formulations:

$$
d_{i}=d_{r}-d_{d}=L_{i}-D_{i}
$$

This definition is extended to the interferometric delay components, $d_{i}=d_{i}^{a}+d_{i}^{g}$ :

$$
\begin{aligned}
d_{i}^{a} & =d_{r}^{a}-d_{d}^{a}=L_{i}-R_{i} \\
d_{i}^{g} & =d_{r}^{g}-d_{d}^{g}=R_{i}-D_{i}
\end{aligned}
$$

\section{B. Atmospheric Raytracing}

The radio length normally is calculated numerically based on rigorous raytracing. The bent raypath is determined by solving the Eikonal equation [14]:

$$
\frac{\partial}{\partial l}(n \hat{\boldsymbol{t}})=\boldsymbol{\nabla} n
$$

where $\hat{\boldsymbol{t}}=\partial \boldsymbol{r} / \partial l$ is the ray tangent direction (a unit vector), $\boldsymbol{r}$ is the evolving ray vector position, $l$ is the incremental arc length, and $\nabla n=\nabla N$ is the spatial gradient of index of refraction or of refractivity. A two-point BVP defines the direct raypath between the satellite and antenna position vectors $\left(\boldsymbol{r}_{\text {sat }} \boldsymbol{r}_{\text {ant }}\right)$; a three-point BVP defines the reflection, which includes additionally the refracted reflection point $\left(\boldsymbol{r}_{\text {sat }}, \boldsymbol{r}_{\text {ant }}, \boldsymbol{r}_{\text {sfc }}^{\prime}\right)$ [5].

The direct satellite elevation angle bending $\delta e_{d}=e_{d}^{\prime}-e$ is the difference between refracted and vacuum satellite elevation angles. It can also be calculated as $\delta e_{d}=\operatorname{acos}\left(\Delta \hat{\boldsymbol{r}}_{\text {sat }}^{\prime} \cdot \Delta \hat{\boldsymbol{r}}_{\text {sat }}\right)$, in terms of the bent ray tangent direction at the receiving antenna, $\Delta \hat{\boldsymbol{r}}_{\text {sat }}^{\prime}=\left(\boldsymbol{r}_{\text {sat }}^{\prime}-\boldsymbol{r}_{\text {ant }}\right) /\left\|\boldsymbol{r}_{\text {sat }}^{\prime}-\boldsymbol{r}_{\text {ant }}\right\|$, in comparison to the geometric or vacuum satellite direction $\Delta \hat{\boldsymbol{r}}_{\text {sat }}=$ $\left(\boldsymbol{r}_{\text {sat }}-\boldsymbol{r}_{\text {ant }}\right) /\left\|\boldsymbol{r}_{\text {sat }}-\boldsymbol{r}_{\text {ant }}\right\|$. A similar reflection satellite elevation bending $\delta e_{r}$ can be defined at the refracted specular point. Their difference $\delta e_{i}=\delta e_{r}-\delta e_{d}$ is the interferometric elevation bending, which may be negligible, provided the reflector height is sufficiently small and the satellite elevation is sufficiently large [5].

Under certain conditions, each direct and reflected bent ray paths may be approximated by straight line segments at the respective refracted or apparent tangent directions:

$$
\boldsymbol{r}=\check{\boldsymbol{r}}+s \cdot \hat{\boldsymbol{t}}
$$

where $\check{\boldsymbol{r}}$ is the initial ray position and $s$ is the incremental ray distance [15]. This approach requires solving only the two-point $\mathrm{BVP}$, for direct rigorous raytracing, to obtain the elevation bending. The three-point BVP may be avoided, being replaced for a rectilinear reflection raytracing. Finally, the direct path is retraced, also based on a rectilinear ray path, for consistency [15]. A remarkable finding was that the combined rectilinear interferometric delay has good accuracy, despite each direct and reflected rectilinear delays having poor accuracy separately. This model accounts for the bulk of angular refraction (elevation bending) and for practically all linear refraction (speed retardation). For further details, the reader is referred to [15].

\section{Zenithal/Slant Factorization}

The zenith interferometric atmospheric delay is defined as:

$$
\begin{aligned}
& d_{i}^{Z}=d_{r}^{Z}-d_{d}^{Z}=2\left(d_{\mathrm{sfc}}^{Z}-d_{\mathrm{ant}}^{Z}\right) \\
& d_{i}^{Z}=2 \int_{r_{\mathrm{sfc}}^{Z}}^{r_{\mathrm{ant}}} N d l=2 \int_{0}^{H} N d H
\end{aligned}
$$

The vector $\boldsymbol{r}_{\mathrm{sfc}}^{Z}$ refers to the surface position immediately under the antenna. Now we can factor out the interferometric slant factor:

$$
f_{i}=d_{i} / d_{i}^{z}
$$

which is responsible for mapping the zenith delay to a specific satellite elevation angle. Analogous expressions exist for the delay components, $f_{i}^{a}=d_{i}^{a} / d_{i}^{z}$ and $f_{i}^{g}=d_{i}^{g} / d_{i}^{z}$ [15]. 


\section{Altimetry Retrieval}

The altimetry retrieval technique employed by a particular instrument will dictate the corresponding atmospheric altimetry bias and correction. One definition follows from half the rate of change of atmospheric delay with respect to the sine of the elevation angle [5]:

$$
\Delta H=-0.5 \partial d_{i} / \partial \sin e=-0.5 d_{i}^{z} \cdot \partial f / \partial \sin e
$$

Equivalently, it can be expressed as the product of zenith delay and half the rate of change of the slant factor. The partial derivatives may be evaluated numerically via finite differencing given a series of delay values versus elevation angle. This expression applies to many retrieval algorithms that utilize the "phase stopping" approach [11], including interferometric Doppler measurements [16] and multipath signal-to-noise ratio (SNR) measurements [1].

For completeness, we mention the "phase anchoring" altimetry approach [11], which requires ambiguity-fixed carrier-phase observables [17], [18]. The corresponding atmospheric altimetry correction follows simply from the absolute ratio $\Delta H=-0.5 d_{i} / \sin e$. Here we focus on the rateof-change formulation, eq.(11).

\section{CLOSED-FORM EXPRESSIONS}

We start highlighting the role of the bottom atmospheric layer, between the receiving antenna and the reflecting surface. The layer thickness equals the antenna height or reflector depth, $H$. It complements the portion of the atmosphere above the antenna, responsible for the elevation bending $\delta e$. Based on these principles, we report the closed-form expressions, denoted with an overhead tilde for distinction from the rigorous results. They are provided first for the atmospheric delay components and later for the corresponding slant factors and altimetry corrections. We end this section complementing the main formulas, for delay and for altimetry, with formulas for uncertainty propagation and also for the elevation angle correction.

\section{A. Atmospheric Layering}

The zenith interferometric delay can be rewritten as $d_{i}^{Z}=$ $2 H N_{\ell}$, where the newly introduced quantity

$$
N_{\ell}=0.5 d_{i}^{z} / H
$$

is the average layer refractivity. Assuming a thin atmospheric layer, it can be approximated as $N_{\ell} \approx\left(N_{\mathrm{sfc}}+N_{\mathrm{ant}}\right) / 2$, in terms of the refractivity at the surface and at the antenna height, or simply $N_{\ell} \approx N_{\text {ant }}$, for negligible $H$.

To further pave the way for the development of closed-form expressions, we define the layer slant distance:

$$
D_{\ell}=2\left\|\boldsymbol{r}_{\mathrm{sfc}}-\boldsymbol{r}_{\mathrm{ant}}\right\|
$$

It is twice the slant distance from antenna to the surface specular reflection point. Besides the vacuum version in eq.(13), a refracted or apparent layer slant distance can also be defined as $D_{\ell}^{\prime}=2\left\|\boldsymbol{r}_{\mathrm{sfc}}^{\prime}-\boldsymbol{r}_{\mathrm{ant}}\right\|$, based on the refracted reflection point, $\boldsymbol{r}_{\mathrm{sfc}}^{\prime}$.

\section{B. Interferometric Atmospheric Delay}

While the general raytraced vacuum distances (ordinary $D$ eq.(13) and apparent $D^{\prime}$ ) are evaluated based on their defining vector norms, the specific closed-form quantities below will be based on trigonometric functions. Furthermore, the closed formulas are only valid for the conditions postulated, i.e., they are derived based on certain assumptions. For example, we assume a flat and horizontal reflecting surface at a small depth $H$ below the antenna (Fig. 1). A symmetric configuration is established, where the direct and reflected angles of incidence are equal.

The closed-form interferometric vacuum distance is wellknown to be:

$$
\widetilde{D}_{i}=2 H \sin e
$$

By analogy, the closed-form apparent interferometric vacuum distance is:

$$
\widetilde{D}_{i}^{\prime}=2 H \sin e^{\prime}
$$

It is based on the apparent elevation angle $e^{\prime} \approx e_{d}^{\prime}$, here approximated as that experienced along the direct path.

Given the quantities above, the closed-form interferometric atmospheric geometric delay is simply the difference between the two previous quantities:

$$
\begin{aligned}
\tilde{d}_{i}^{g}=\widetilde{D}_{i}^{\prime}-\widetilde{D}_{i}= & 2 H\left(\sin e^{\prime}-\sin e\right) \\
& \cong 2 H \delta e \cos e
\end{aligned}
$$

This formulation is based on the assumption of a far source (transmitting satellite), so that direct and reflected raypaths arrive parallel. It was first given explicitly by [4]. It is proportional to reflector height $H$, so it is not necessarily negligible, unless the elevation bending $\delta e$ is also small. The approximation on the right-hand side is derived from the anglesum and small-angle trigonometric identities and requires $\delta e$ to be expressed in radians.

Next, we define the closed-form apparent layer slant distance:

$$
\widetilde{D}_{\ell}^{\prime}=2 H / \sin e^{\prime}
$$

The closed-form interferometric atmospheric along-path delay is then postulated to be simply a scaled version of the apparent layer slant distance:

$$
\tilde{d}_{i}^{a}=N_{\ell} \widetilde{D}_{\ell}^{\prime}=d_{i}^{z} / \sin e^{\prime} \cong 2 H N_{\ell} \csc e^{\prime}
$$

This formulation is based on the assumption of a vertically stratified atmosphere, so that the along-path delay in the portion of the atmosphere above the antenna cancels out between reflected and direct raypaths. This is the only term normally considered in GNSS-R, with the cosecant factor sometimes replaced by a direct slant factor, $f_{d}$, as derived from mapping functions developed for GNSS positioning. Notice that term is directly proportional to reflector height $H$ while it depends indirectly on the station altitude via the average layer refractivity $N_{\ell}$.

Finally, the closed-form total interferometric atmospheric delay is simply the sum of its two components (eq. 16,18):

$$
\tilde{d}_{i}=\tilde{d}_{i}^{a}+\tilde{d}_{i}^{g}=2 H N_{\ell} \csc e^{\prime}+2 H \delta e \cos e
$$

This two-term total is not previously found in the literature. For completeness, we also give the closed-form interferometric radio length:

$$
\tilde{L}_{i}=\widetilde{D}_{i}+\tilde{d}_{i}=2 H\left(\sin e^{\prime}+N_{\ell} / \sin e^{\prime}\right)
$$

It should be emphasized that the ancillary atmospheric parameters (average refractivity $N_{\ell}$ and elevation bending $\delta e$ ) will be taken from a previous direct rigorous raytracing, as our goal is to validate the mathematical formulation of the closed expressions. Alternatively, in case raytracing software is not available, empirical models such as the Global Pressure and 
Temperature (GPT) [19], [20] can be used to obtain the refractivity at the station and models such as those of [21], [22] can be used to approximate the elevation bending. In this case, the discrepancy between closed formulas and raytracing will include also errors in the atmospheric proxies $\left(N_{\ell}\right.$ and $\delta e$ ).

\section{Interferometric Slant Factors}

Slant factors cancel out the effect of zenith delay, isolating the elevation angle dependency. For example, the closed-form interferometric atmospheric along-path slant factor reads:

$$
\tilde{f}_{i}^{a}=\frac{\tilde{d}_{i}^{a}}{d_{i}^{z}}=\frac{1}{\sin e^{\prime}}=\csc e^{\prime}
$$

Analogously, for the closed-form interferometric atmospheric geometric slant factor we can write:

$$
\tilde{f}_{i}^{g}=\frac{\tilde{d}_{i}^{g}}{d_{i}^{z}}=\frac{\sin e^{\prime}-\sin e}{N_{\ell}} \cong \frac{\delta e \cos e}{N_{\ell}}
$$

The expression for the closed-form interferometric total slant factor is:

$$
\tilde{f}_{i}=\tilde{f}_{i}^{a}+\tilde{f}_{i}^{g}=\csc e^{\prime}+\delta e \cos e / N_{\ell}
$$

These trigonometric approximations will be compared to their raytraced values, $f_{i}$, obtained independently following their defining numerical ratios (eq.10).

\section{Atmospheric Altimetry Correction}

We provide the closed-form relative atmospheric altimetry correction, normalized by reflector height $H$, to emphasize the linear dependence on $H$. For rate-of-change altimetry retrievals, we have:

$$
\begin{gathered}
\frac{\Delta \widetilde{H}_{i}^{a}}{H}=\frac{N_{\ell}}{\sin ^{2} e^{\prime}}(1+\xi) \approx N_{\ell} \csc ^{2} e^{\prime} \\
\frac{\Delta \widetilde{H}_{i}^{g}}{H}=-\delta e \tan e(1+\xi)+\xi \approx \xi \\
\xi=\partial \delta e / \partial e
\end{gathered}
$$

These expressions are not previously found in the literature. It is evident the dependence on elevation bending $\delta e$ (which must be expressed in radians when used as multiplicative factor) as well as its rate of change with respect to geometric elevation angle, $\xi$. These trigonometric approximations will be compared to their raytraced values, $\Delta H_{i}$, obtained independently via numerical derivative of delays as per eq.(11).

\section{E. Altimetry Uncertainty Propagation}

The closed-form atmospheric altimetry corrections (eq.2325) allow us to perform, for the first time, uncertainty propagation on their input parameters:

$$
\begin{gathered}
\sigma_{\Delta \widetilde{H}_{i}^{a} / H} \approx \sigma_{N_{\ell}} \cdot \csc ^{2} e^{\prime} \\
\sigma_{\Delta \widetilde{H}_{i}^{g} / H} \approx \sigma_{\xi}
\end{gathered}
$$

It is evident that the uncertainty in the atmospheric altimetry correction due to that of refractivity, $\sigma_{N_{\ell}}$, increases with decreasing elevation angle in its along-path component, $\Delta \widetilde{H}_{i}^{a}$. On the other hand, the uncertainty in the geometric altimetry component $\Delta H_{i}^{g}$ is driven essentially by that of the rate-ofchange of elevation bending with respect to the elevation angle, $\sigma_{\xi}$. Applying a linear approximation, we find:

$$
\begin{aligned}
\xi & =\lim _{\Delta e \rightarrow 0} \frac{\Delta \delta e}{\Delta e} \approx \frac{\delta e_{2}-\delta e_{1}}{e_{2}-e_{1}} \\
\sigma_{\xi} & =\sigma_{\delta e} \sqrt{2\left(1-\rho_{\delta e}\right)}|\Delta e|^{-1}
\end{aligned}
$$

Thus, $\sigma_{\xi}$ is driven by the uncertainty of elevation bending $\sigma_{\delta e}$ (in radians), its autocorrelation $\rho_{\delta e}$ (between -1 and +1 ), and the elevation range $\Delta e$ under which $\xi$ is evaluated (e.g., $e \pm 1^{\circ}$ ). For the case of uncorrelated elevation bending noise $\left(\rho_{\delta e}=0\right)$, we find $\sigma_{\xi}=\sigma_{\delta e} /|\Delta e|$. So, uncertainty propagation advises to maximize the elevation angle range $\Delta e$ to minimize the altimetry uncertainty, $\sigma_{\Delta \widetilde{H}_{i}}$.

\section{F. Atmospheric Elevation Angle Correction}

GNSS-R altimetry software may rely only on modifying the input satellite elevation angle to compensate for atmospheric refraction, with no possibility of using the total atmospheric interferometric delay explicitly (eq.19). We thus define an effective satellite elevation angle $e^{*}=e+\delta e^{*}$ such that, when input to the ordinary vacuum delay formula (eq.14), produces the total radio length (eq.20):30

$$
e^{*}:\left.D_{i}\right|_{e^{*}}=2 H \sin e^{*}=L_{i}
$$

Isolating the atmospheric elevation angle correction $\delta e^{*}=$ $e^{*}-e$, we find:

$$
\delta e^{*}=\operatorname{asin}\left(\sin e^{\prime}+N_{\ell} / \sin e^{\prime}\right)-e
$$

Applying the angle-sum and small-angle trigonometric identities, it can be approximated by the sum of elevation bending $\delta e$ and a second-order elevation correction:

$$
\delta e^{*}=\delta e+N_{\ell} /(\sin e \cos e)
$$

where the result is expressed in radians.

We provide the complete formula (eq.31) for the first time in the literature. It should be noted that the usage of [6] corresponds to the elevation bending only $\left(\delta e^{*} \cong \delta e\right.$ ), while the usage of [23] seems equivalent to the second term only $\left(\delta e^{*} \cong N_{\ell} /(\sin e \cos e)\right)$ of eq. (31).

\section{NUMERICAL RESULTS AND DISCUSSION}

In the following, we assess closed-form expressions developed above, by comparison to raytracing results. We start with rectilinear raytracing then justify the discrepancies by juxtaposing the rigorous raytracing results.

As atmospheric model, we used the CIRA climatology [24], which neglects humidity. A single vertical profile is extracted at zero latitude and longitude and used to structure a spherically osculating atmosphere [25]. Geographical and temporal variations are outside the scope of this work. The transmitter satellite is at GNSS orbital altitude $(\sim 20,000 \mathrm{~km})$. A fixed $10-$ $\mathrm{m}$ reflector height is employed throughout, as a value representative of ground-based GNSS-R stations.

For completeness, we also include an ad-hoc model introduced in [9] and assessed by [4]:

$$
\tilde{d}_{i}^{*}=d_{i}^{z} \cdot f_{d}
$$

where $f_{d}=d_{d} / d_{d}^{z}$ is the direct slant factor, obtained from rigorous direct raytracing. This model is motivated by the use in GNSS-R of mapping functions developed for GNSS positioning. The corresponding altimetry correction $\Delta \widetilde{H}_{i}^{*}=$ $0.5 \partial \tilde{d}_{i}^{*} / \partial \sin e$, is obtained via numerical differentiation. The same numerical approach is used to obtain altimetry corrections from the raytracing delay results.

\section{A. Atmospheric Delay}

We observe in Fig. 2 (top) the overall excellent agreement between closed-form total delay $\tilde{d}_{i}$ and the respective 
rectilinear raytracing result, whose discrepancy is shown in the bottom panel of Fig. 2. For the atmospheric along-path delay $\tilde{d}_{i}^{a}$, the agreement is nearly exact at high elevations and it remains at the millimeter level at low elevations, where the discrepancy grows exponentially, reaching $3 \mathrm{~mm}$ or $7 \%$ near the horizon. The atmospheric geometric delay, $\tilde{d}_{i}^{g}$ has much better agreement with raytracing results throughout the whole elevation angle domain.

Although we have used the same elevation bending $\delta e$ as input for both closed formulas and rectilinear raytracing, the agreements is not exact. To investigate the source of this small disagreement, we have temporarily changed the raytracing settings to force a more perfect matching with the assumptions behind the closed formulas. More specifically, we have increased the orbital altitude by $100 x$ to put the transmitter more distant and replaced the radial stratification for a plane-tangent atmospheric structure. Although not realistic for GNSS-R practice, such settings do improve the agreement of closedformulas to better than $0.01 \mathrm{~mm}$ (figure not shown). Therefore, there is room for further improvements in the closed formulas in the future.

The ad-hoc formulation, $\tilde{d}_{i}^{*}$ (eq.32), follows closely the rectilinear along-path results; their discrepancy, shown in Fig. 3 (bottom), is similar to that between $\tilde{d}_{i}^{a}$ and the respective rectilinear component, albeit with reversed sign and slightly smaller magnitude. The discrepancy is larger between $\tilde{d}_{i}^{*}$ and the total rectilinear delay, reaching $5 \mathrm{~cm}$ at the lowest elevation angle. Thus, the ad-hoc model based on direct raytracing and similar mapping functions developed for GNSS positioning is seen not to be an adequate replacement for the total interferometric atmospheric delay.

Fig. S1 (in the supplementary material) recapitulates the discrepancy in interferometric atmospheric delay between rectilinear raytracing and rigorous raytracing, as checked originally in [12]. The agreement in terms of total delay $\tilde{d}_{i}$ and its components is one order of magnitude smaller than that shown in Fig. 2. That means it is safe to compare closed formulas to rectilinear results as a surrogate for rigorous results.

\section{B. Slant Factors}

In Fig. 3 (top), the total slant factors (eq.10) and their components are presented. There is excellent agreement between closed-form and the respective rectilinear results, whose discrepancy is shown in the bottom panel of the same figure.

For the along-path slant factor, $\tilde{f}_{i}^{a}$, the agreement is nearly exact at high elevations and it remains at the millimeter level at low elevations for a $5 \mathrm{~mm}$ nominal interferometric zenith delay $\left(d_{i}^{z}=5 \mathrm{~mm} \approx 2 \cdot 10 \mathrm{~m} \cdot 250 \cdot 10^{-6}\right)$; near the horizon, the discrepancy in $\tilde{f}_{i}^{a}$ grows exponentially, reaching $6.5 \%$. The atmospheric geometric slant factor, $\tilde{f}_{i}^{g}$, has near exact agreement with the rectilinear raytracing result, for all elevation angles. For the total slant factor $\tilde{f}_{i}$, its discrepancy is dominated by that in $\tilde{f}_{i}^{a}$, as the discrepancy in $\tilde{f}_{i}^{g}$ is negligible. Finally, the direct slant factor, $f_{d}$, follows closely the rectilinear along-path result (Fig. 3, top). In Fig. 3 (bottom), their discrepancy is similar to that of $\tilde{f}_{i}^{a}$, albeit with reversed sign and slightly smaller magnitude. Thus, if $f_{d}$ were to be used to compute the total delay, the error would reach almost $40 \mathrm{~cm} / \mathrm{m}$ at the lowest elevation angle (Fig. 3, inset).

Fig. S2 highlights that interferometric along-path atmospheric delay $d_{i}^{a}$, driven by the apparent layer slant distance $D_{\ell}$ (eq.18), is unrelated to the interferometric vacuum distance $D_{i}$, whose self-difference drives the geometric atmospheric delay $d_{i}^{g}$ (eq.16). With respect to the trigonometric factors $\csc e^{\prime}$ and $\delta e \cos e$ involved in, respectively, $\tilde{d}_{i}^{a}$ and $\tilde{d}_{i}^{g}$, it is interesting to see the similarity in their graphs (Fig. 3, top), despite having very different formulas. It is also worth noticing that although the trigonometric elements differ greatly in order of magnitude, this is compensated to some extent by the refractivity value $\left(N_{\ell} \cong 0.00025\right)$. The ratio of the two closed-form delay components reads:

$$
\frac{\tilde{d}_{i}^{g}}{\tilde{d}_{i}^{a}}=\frac{\delta e \cos e \sin e^{\prime}}{N_{\ell}}
$$

It attains almost unity at 10-degree elevation (Fig. S2, left-hand axis) where the two delay components are mostly balanced. Also, for the conditions considered, $\tilde{d}_{i}^{a}$ is always greater than $\tilde{d}_{i}^{g}$.

\section{Atmospheric Altimetry Correction}

Now turning to the main result of interest, the atmospheric altimetry correction (eq.23-24), we show it normalized by reflector height, in units of centimeters per meter in Fig. 4 (top). For example, for a satellite at $5^{\circ}$ elevation angle, the total relative atmospheric altimetry correction $\Delta \widetilde{H}_{i} / H$ amounts to 4.5 $\mathrm{cm} / \mathrm{m}$, which scales to $45 \mathrm{~cm}$ altimetry correction for a reflector height of $10 \mathrm{~m}$; on the other hand, near zenith, the correction amounts to $0.8 \mathrm{~mm} / \mathrm{m}$ or $8 \mathrm{~mm}$ for a 10 -m reflector height.

The inset in Fig. 4 (top) emphasizes that the geometric altimetry correction component (eq.24) converges to a constant value $(0.5 \mathrm{~mm} / \mathrm{m})$ at zenith, despite the respective geometric delay component (eq.16) converging to zero. This is explained by the rate of change of elevation bending with respect to elevation angle (eq.25).

The agreement between closed-form and rectilinear results (Fig. 4, bottom) is good for the along-path altimetry component $\Delta \widetilde{H}_{i}^{a}$, whose discrepancy remains smaller than a tenth of the correction. The agreement is excellent for the geometric atmospheric altimetry correction, $\Delta \widetilde{H}_{i}^{g}$, whose discrepancy is negligible for practical purposes. The closed-form total atmospheric altimetry correction follows closely the raytracing results; the corresponding discrepancy $\Delta \widetilde{H}_{i}$ is dominated by the discrepancy in the along-path component which remains less than $5 \mathrm{~mm} / \mathrm{m}$ even at the low elevation angles. The ad-hoc model, $\Delta \widetilde{H}_{i}^{*}$, agrees with the along-path term and misses the geometric contribution.

The ratio of the respective altimetry correction components:

$$
\frac{\Delta \widetilde{H}_{i}^{g}}{\Delta \widetilde{H}_{i}^{a}}=\frac{\xi}{N_{\ell} / \sin ^{2} e^{\prime}}
$$

is also shown in Fig. S3 (right-hand axis) and behaves in the opposite way to the delay ratio. The geometric component is larger than the along-path one for most of the elevation angle domain. The ratio is balanced at $12^{\circ}$ satellite elevation angle due to the rate of change with respect to the elevation angle. Near zenith, although the absolute altimetry correction is minimal, the ratio approaches its maximum; at $85^{\circ}$ satellite 
elevation angle, the geometric altimetry correction is almost twice the along-path one.

\section{Cutoff Elevation Angle}

We investigate the limiting conditions for negligible atmospheric altimetry correction by showing in Fig. 5 its contour lines over a bivariate domain of satellite elevation angle and reflector height. The plot can be interpreted following vertical or horizontal lines for either a fixed reflector height or a fixed elevation angle.

For instance, when reflector height equals $1 \mathrm{~m}$, a $13^{\circ}$ cut-off elevation angle corresponds to a negligible correction, of less than $1 \mathrm{~cm}$. If a greater reflector height is utilized, the cutoff elevation angle grows almost linearly, e.g., it is $19^{\circ}$ at a $2-m$ reflector height and $30^{\circ}$ at $5 \mathrm{~m}$. For an antenna located an altitude of $50 \mathrm{~m}$ above the reflecting surface, the altimetry correction is at least $10 \mathrm{~cm}$ for any satellite observed above $30^{\circ}$ elevation angle; for that height, applying nothing but a tighter elevation cut-off angle of $55^{\circ}$ would reduce the altimetry error to $5 \mathrm{~cm}$.

From an orthogonal point of view, the same satellite would produce an altimetry error proportional to the reflector height at which is observed. For example, an error of nearly $0.1 \mathrm{~cm}$ and $10 \mathrm{~cm}$ correspond to a satellite at $30^{\circ}$ elevation angle when observed from antennas located at approximately $0.5 \mathrm{~m}$ and 50 $\mathrm{m}$ above the surface, respectively.

Fig. 5 (bottom) highlights the contour for $1-\mathrm{cm}$ altimetry correction and its components. It is apparent that heights greater than $12.5 \mathrm{~m}$ need altimetry correction, even near zenith. Conversely, at 5-degree elevation angle, even a few tens of $\mathrm{cm}$ of height are enough to cause a significant altimetry correction.

\section{E. Atmospheric Elevation Angle Correction}

The atmospheric elevation correction $\delta e^{*}$ (eq.30), resembles twice the interferometric bending $\delta e=e^{\prime}-e$ yet offset by approximately $50 \%$ throughout the elevation angle domain (Fig. 6). Specifically, $\delta e^{*}$ approaches $\delta e$ the closest at $45^{\circ}$ elevation while it diverges at the edges i.e., at satellite elevation angles of $5^{\circ}$ and $85^{\circ}$, their difference is $0.16^{\circ}$ and $0.18^{\circ}$ respectively. The atmospheric elevation correction is especially pronounced near zenith, where it needs to account for the alongpath delay, in addition to the elevation bending.

\section{CONCLUSIONS}

In this study, we derived from first principles and validated closed-form expressions for the interferometric atmospheric delay, assuming a flat and horizontal reflecting surface at a small depth below the receiving antenna. We also provided closed-form atmospheric altimetry correction expressions, necessary for unbiased GNSS-R sea-level retrievals.

Firstly, we described and formulated the intrinsic propagation quantities based on two geometric quantities: satellite elevation angle and reflector height. A greater insight into the physics of the problem was given by considering the atmospheric layer between antenna and surface. This revealed that even small reflector heights are still subject to the atmospheric refraction originating above the receiving antenna.

Next, the closed-form expression for the interferometric along-path delay was given as a function of the layer slant distance and the mean refractivity. The interferometric geometric delay was computed as the difference between the refracted and unrefracted interferometric distances. The summation of the two yielded the total delay.

We then extended the delays' definitions to the respective slant factors by factoring out the zenith delay. The corresponding atmospheric altimetry corrections were derived via analytical formulas and approximations thereof. We gave a synopsis of the uncertainty propagation in the atmospheric altimetry correction. Finally, we presented the elevation correction that can be utilized to account for the total atmospheric delay, enabling easy adaptation in existing GNSS-R software.

We assessed the closed-form expressions against rigorous and rectilinear raytracing results. The interferometric geometric atmospheric delay exhibited excellent agreement, with negligible discrepancies compared to raytracing. The interferometric along-path atmospheric delay showed good agreement to raytracing, with discrepancies due to the refractivity approximation, that grew towards the horizon but remained within few mm. Finally, an ad-hoc model based on direct propagation proved insufficient to predict the total delay.

Slant factors showed similar performance to the delays results and the discussion on their trigonometric elements highlighted the similarity of the two components latter albeit their differences in scale. The corresponding atmospheric altimetry corrections were similarly derived and validated, revealing a linear dependence on reflector height. Their assessment against raytracing followed similar conclusions as for the atmospheric delays.

We then presented the limiting conditions for neglecting any atmospheric altimetry correction, in terms of a cutoff satellite elevation for variable reflector height. For example, at a reflector height of $1 \mathrm{~m}$ the cut-off elevation angle should be $13^{\circ}$ to keep the altimetry correction below $1 \mathrm{~cm}$. The same $1-\mathrm{cm}$ altimetry correction would impose a cut-off elevation angle of $30^{\circ}$ if observed from a 5-m reflector height. In any case, these cutoffs can be surpassed with the application of the correction expressions here developed.

It should be highlighted that the closed-form expressions need no reflection raytracing, only line-of-sight raytracing so as to obtain the elevation bending and mean refractivity as input. Alternatively, existing tabulations and/or empirical models of elevation bending and mean refractivity may be used by GNSS$\mathrm{R}$ users with no access to raytracing software.

Many neglected effects also to be considered in the future, such as: atmospheric humidity (instead of just dry gases); curvature of the Earth (spherical reflecting surface instead of a tangent plane); geographical variations (station latitude, longitude, altitude); temporal variations (time of day, day of year, year-to-year); greater reflector height variations (thicker atmospheric layer); and directional variations (satellite azimuth). For further discussion of systematic and random error sources, the reader is referred to Nikolaidou (2020). 


\section{References}

[1] K. M. Larson, R. D. Ray, F. G. Nievinski, and J. T. Freymueller, "The Accidental Tide Gauge: A GPS Reflection Case Study From Kachemak Bay, Alaska," vol. 10, no. 5, pp. 1200-1204, Sep. 2013.

[2] K. M. Larson, R. D. Ray, S. D. P. Williams, K. M. Larson, R. D. Ray, and S. D. P. Williams, "A 10-Year Comparison of Water Levels Measured with a Geodetic GPS Receiver versus a Conventional Tide Gauge," J. Atmos. Ocean. Technol., vol. 34, no. 2, pp. 295-307, Feb. 2017.

[3] F. Geremia-Nievinski et al., "SNR-based GNSS reflectometry for coastal sea-level altimetry - Results from the first IAG intercomparison campaign," J. Geod., vol. 94, no. 8, p. 70, Aug. 2020.

[4] S. D. P. Williams and F. G. Nievinski, "Tropospheric delays in ground-based GNSS multipath reflectometry-Experimental evidence from coastal sites," J. Geophys. Res. Solid Earth, vol. 122, no. 3, pp. 2310-2327, Mar. 2017.

[5] T. Nikolaidou, C. M. Santos, D. P. S. Williams, and F. GeremiaNievinski, "Raytracing atmospheric delays in ground-based GNSS reflectometry," J. Geod., vol. 94, no. 68, Aug. 2020.

[6] A. Santamaría-Gómez and C. Watson, "Remote leveling of tide gauges using GNSS reflectometry: case study at Spring Bay, Australia," GPS Solut., vol. 21, no. 2, pp. 451-459, Apr. 2017.

[7] F. Fabra et al., "Phase altimetry with dual polarization GNSS-R over sea ice," IEEE Trans. Geosci. Remote Sens., vol. 50, no. 6, pp. 2112 2121, Jun. 2012.

[8] S. Khajeh, A. A. Ardalan, H. Schuh, and H. S. S. Khajeh, A. A. Ardalan, "Interferometric Path Models for GNSS Ground-Based Phase Altimetry S.," in The International Archives of the Photogrammetry, Remote Sensing and Spatial Information Sciences, 2019, vol. XLII-4/W18, p. Volume XLII-4/W18, 2019.

[9] R. N. Treuhaft, S. T. Lowe, C. Zuffada, and Y. Chao, "2-cm GPS altimetry over Crater Lake," Geophys. Res. Lett., vol. 28, no. 23, pp. 4343-4346, Dec. 2001.

[10] E. Cardellach, F. Fabra, O. Nogués-Correig, S. Oliveras, S. Ribó, and A. Rius, "GNSS-R ground-based and airborne campaigns for ocean, land, ice, and snow techniques: Application to the GOLD-RTR data sets," Radio Sci., vol. 46, no. 5, pp. 1-16, Dec. 2011.

[11] V. U. Zavorotny, S. Gleason, E. Cardellach, and A. Camps, "Tutorial on remote sensing using GNSS bistatic radar of opportunity," IEEE Geosci. Remote Sens. Mag., vol. 2, no. 4, pp. 8-45, Dec. 2014.

[12] T. Nikolaidou, M. C. Santos, S. D. P. Williams, and F. GeremiaNievinski, "A simplification of rigorous atmospheric raytracing based on judicious rectilinear paths for near-surface GNSS reflectometry," Earth, Planets Sp., vol. 72, no. 91, Dec. 2020.

[13] V. Nafisi et al., "Comparison of Ray-Tracing Packages for Troposphere Delays," IEEE Trans. Geosci. Remote Sens., vol. 50, no. 2, pp. 469-481, Feb. 2012.

[14] M. Born and E. Wolf, Principles of optics Electromagnetic theory of propagation, interference and diffraction of light, 7th expand. Cambridge University Press, 1999.

[15] T. Nikolaidou, "Atmospheric Delay Modelling for Ground-based GNSS Reflectometry (Ph.D. Dissertation)," University of New Brunswick, 2020.

[16] A. M. Semmling et al., "On the retrieval of the specular reflection in GNSS carrier observations for ocean altimetry," Radio Sci., vol. 47, no. 6, Dec. 2012.

[17] J. S. Löfgren, R. Haas, H.-G. Scherneck, and M. S. Bos, "Three months of local sea level derived from reflected GNSS signals," Radio Sci., vol. 46, no. 6, Dec. 2011.

[18] M. Martin-Neira, P. Colmenarejo, G. Ruffini, and C. Serra, "Altimetry precision of $1 \mathrm{~cm}$ over a pond using the wide-lane carrier phase of GPS reflected signals," Can. J. Remote Sens., vol. 28, no. 3, pp. 394-403, 2002.

[19] K. Lagler, M. Schindelegger, J. Böhm, H. Krásná, and T. Nilsson, "GPT2: Empirical slant delay model for radio space geodetic techniques.," Geophys. Res. Lett., vol. 40, no. 6, pp. 1069-1073, Mar. 2013.

[20] D. Landskron and J. Böhm, "VMF3/GPT3: refined discrete and empirical troposphere mapping functions," J. Geod., vol. 92, no. 4, pp. 349-360, Apr. 2017.

[21] G. G. Bennett, "The Calculation of Astronomical Refraction in Marine Navigation," J. Navig., vol. 35, no. 02, p. 255, May 1982.

[22] T. Hobiger, R. Ichikawa, Y. Koyama, and T. Kondo, "Fast and accurate ray-tracing algorithms for real-time space geodetic applications using numerical weather models," J. Geophys. Res. Atmos., vol. 113, no. 20, pp. 1-14, Oct. 2008.

[23] D. J. Purnell, N. Gomez, N.-H. H. Chan, J. Strandberg, D. M. Holland, and T. Hobiger, "Quantifying the Uncertainty in GroundBased GNSS-Reflectometry Sea Level Measurements," IEEE J. Sel. Top. Appl. Earth Obs. Remote Sens., 2020.

[24] E. L. Fleming, S. Chandra, J. J. Barnett, and M. Corney, "Zonal mean temperature, pressure, zonal wind and geopotential height as functions of latitude," Adv. Sp. Res., vol. 10, no. 12, pp. 11-59, Jan. 1990.

[25] F. G. Nievinski and M. C. Santos, "Ray-tracing options to mitigate the neutral atmosphere delay in GPS," Geomatica, vol. 64, no. 2, pp. 191-207, 2010. 


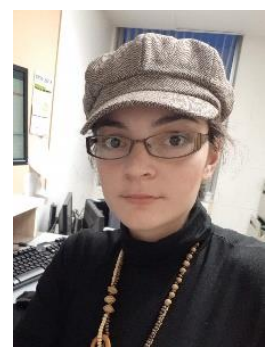

Thalia Nikolaidou received her Diploma in Rural and Surveying Engineering from Aristotle University of Thessaloniki (AUTh), Greece. In 2020, she completed her $\mathrm{Ph} . \mathrm{D}$. on tropospheric imprints on GNSS Reflectometry and advanced modelling of atypical atmospheric conditions for GNSS applications. Her research interests include the use of global and regional Numerical Weather Models for parametrization and precise prediction of the tropospheric delay, modelling the asymmetry of the lower atmosphere and Precise Point Positioning for GNSS-Meteorology. She is associated with the UNB-Vienna Mapping Functions 1 Service (VMF1) and is part of the UNB's GNSS Analysis and Positioning Software (GAPS) developing team.

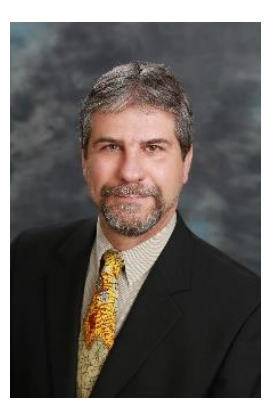

Marcelo C. Santos is a Professor at the Department of Geodesy and Geomatics Engineering at the University of New Brunswick (UNB), Canada. He holds a M. Sc. Degree in Geophysics from the National Observatory, in Rio de Janeiro, and a Ph.D. Degree in Geodesy from the University of New Brunswick. His research interest relates to geodetic applications of global navigation satellite system and to modelling of Earth's gravity

field. His most recent contributions include a rigorous definition of orthometric height, research on precise point positioning (PPP), the development of the PPP research platform GAPS, and investigation on the use of numerical weather prediction models for modelling the neutralatmosphere, including the implementation of a (UNB) VMF1 Service as a contribution to the Global Geodetic Observing System (GGOS). He is a Fellow of the International Association of Geodesy.

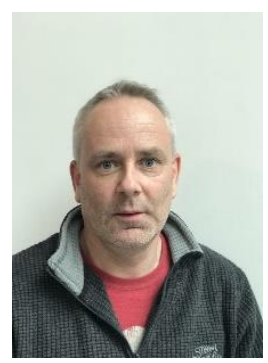

Simon D. P. Williams received the B.Sc degree in Geology and Geophysics from the University of Durham, UK in 1991 and a Ph.D. in Geophysics from the University of Durham, UK in 1995. From 1996 to 1999, he was a Research Assistant at the Scripps Institution of Oceanography, University of California, San Diego, CA, USA. Since 1999, he has been a Senior Researcher in the Sea Level and Ocean Climate Group at the National Oceanography Centre, Liverpool, UK. He is the author of over 50 articles and his research interests include ground based GNSS multipath reflectometry, sea level studies using tide gauges, GNSS and satellite altimetry and stochastic modelling and uncertainty analysis of geophysical time series. He was associate editor for the Journal of Geodesy from 2007 to 2015.

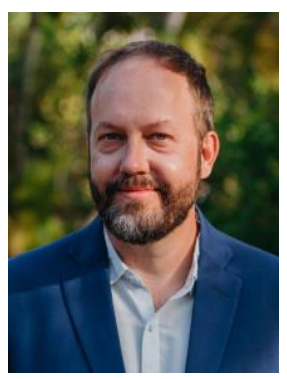

Felipe Geremia-Nievinski received the B.E. from the Federal University of Rio Grande do Sul (UFRGS) in Porto Alegre, RS, Brazil in 2005; the M.Sc.E. (geomatics engineering) from the University of New Brunswick (UNB) in Fredericton, NB, Canada in 2009; and the $\mathrm{Ph} . \mathrm{D}$. degree (aerospace engineering sciences) from the University of Colorado, Boulder, CO, USA in 2013. In 2016 he became a faculty member at UFRGS (Department of Geodesy, Institute of Geosciences), where he also served as director of undergraduate studies in geomatics engineering (2016-2020) and serves as student advisor in the postgraduate program in remote sensing (2017-). His research interests include satellite geodesy, with focus on atmospheric refraction as well as GPS/GNSS reflectometry. He is a fellow of the International Association of Geodesy and associate editor for the Journal of Geodesy. 


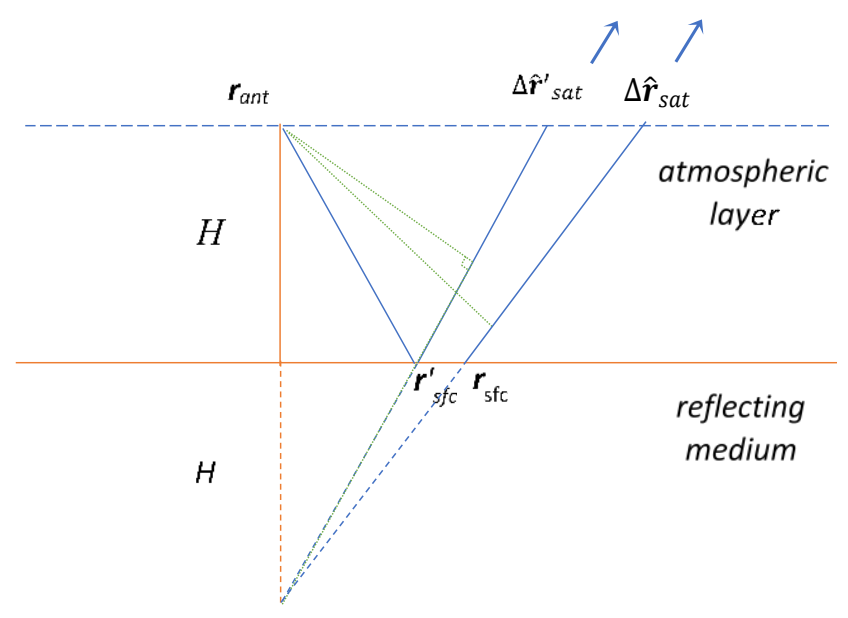

Fig. 1: Refracted notable points and directions of rectilinear raytracing.
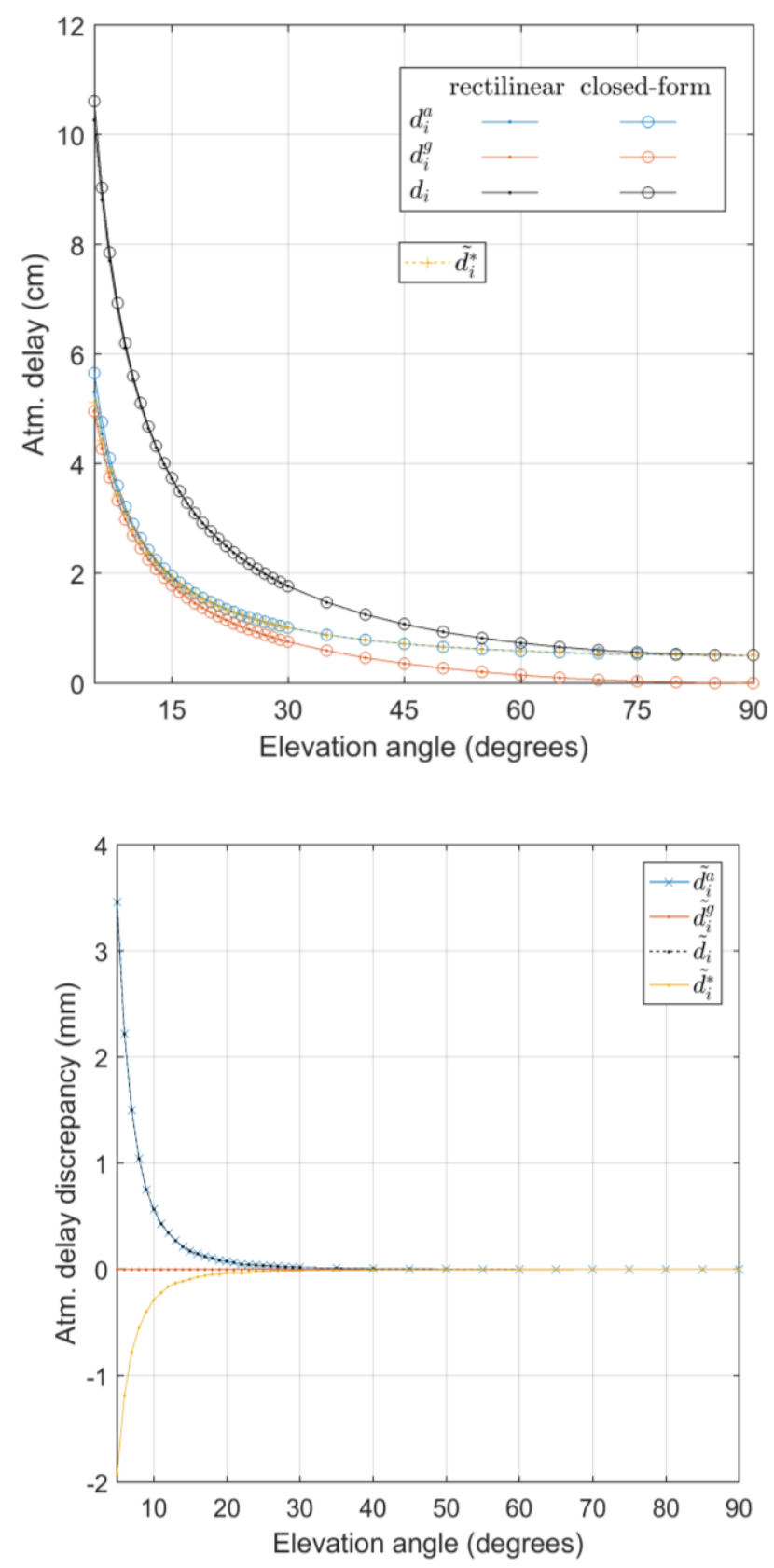

Fig. 2: Closed-form and rectilinear raytracing interferometric atmospheric delay (top) and their discrepancy (bottom) as a function of satellite elevation angle (for a fixed 10-m reflector height). 

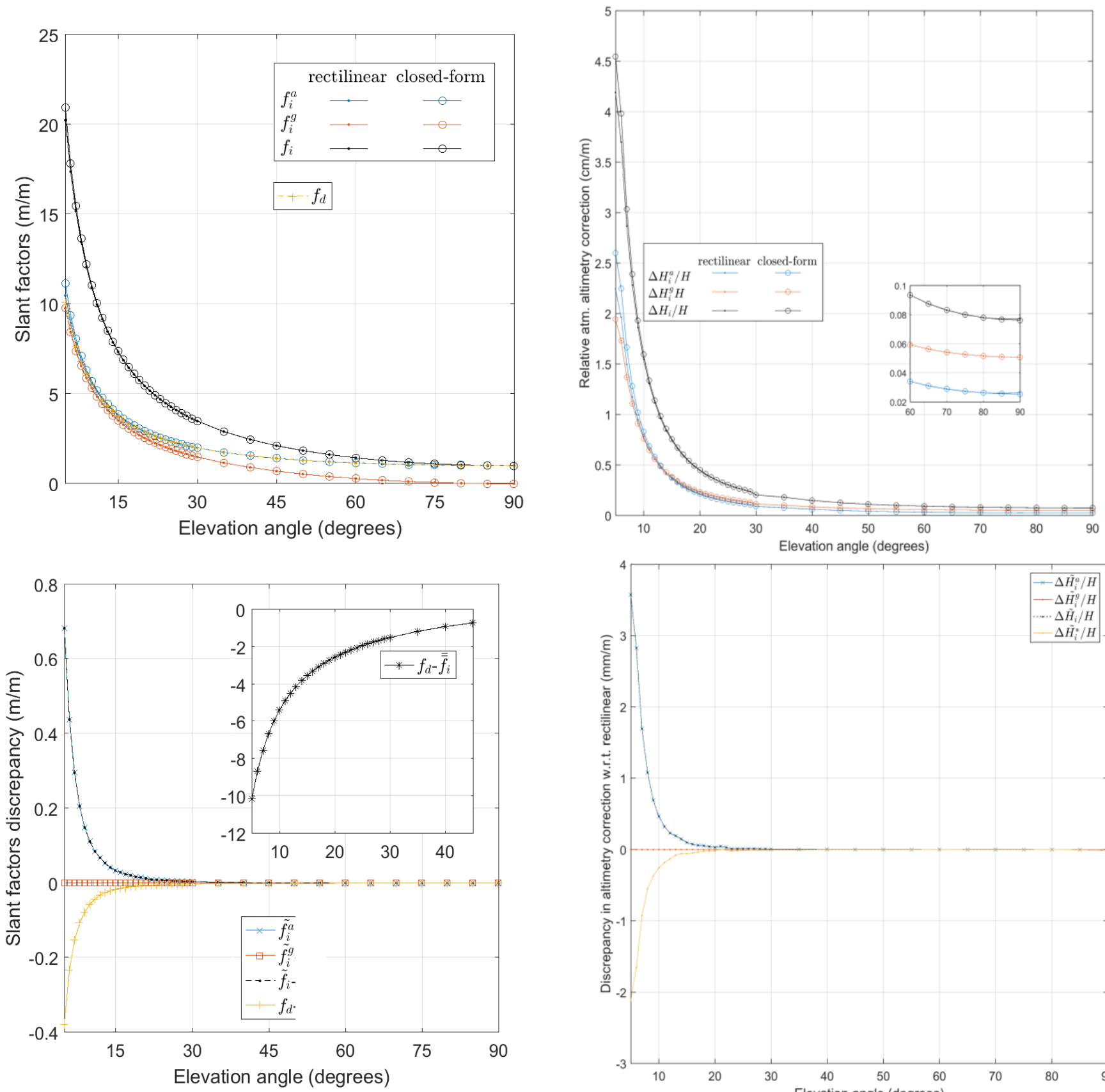

Fig. 3: Interferometric slant factor and components (top) and the discrepancy between closed formulas and rectilinear raytracing (bottom), as a function of satellite elevation angle; the bottom panel inset shows the discrepancy between direct slant factor and the total interferometric slant factor, also in units of $\mathrm{m} / \mathrm{m}$.

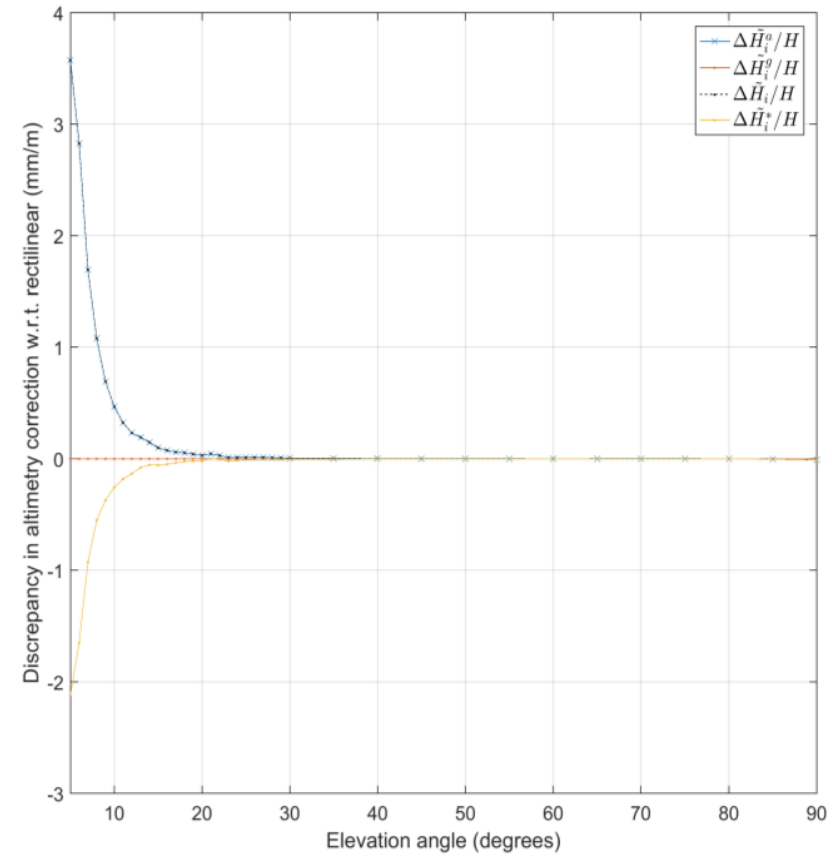

Fig. 4: Relative atmospheric altimetry correction (top) and the discrepancy between closed formulas and rectilinear raytracing (bottom) as a function of satellite elevation angle; the top panel inset highlights the near zenith region. 

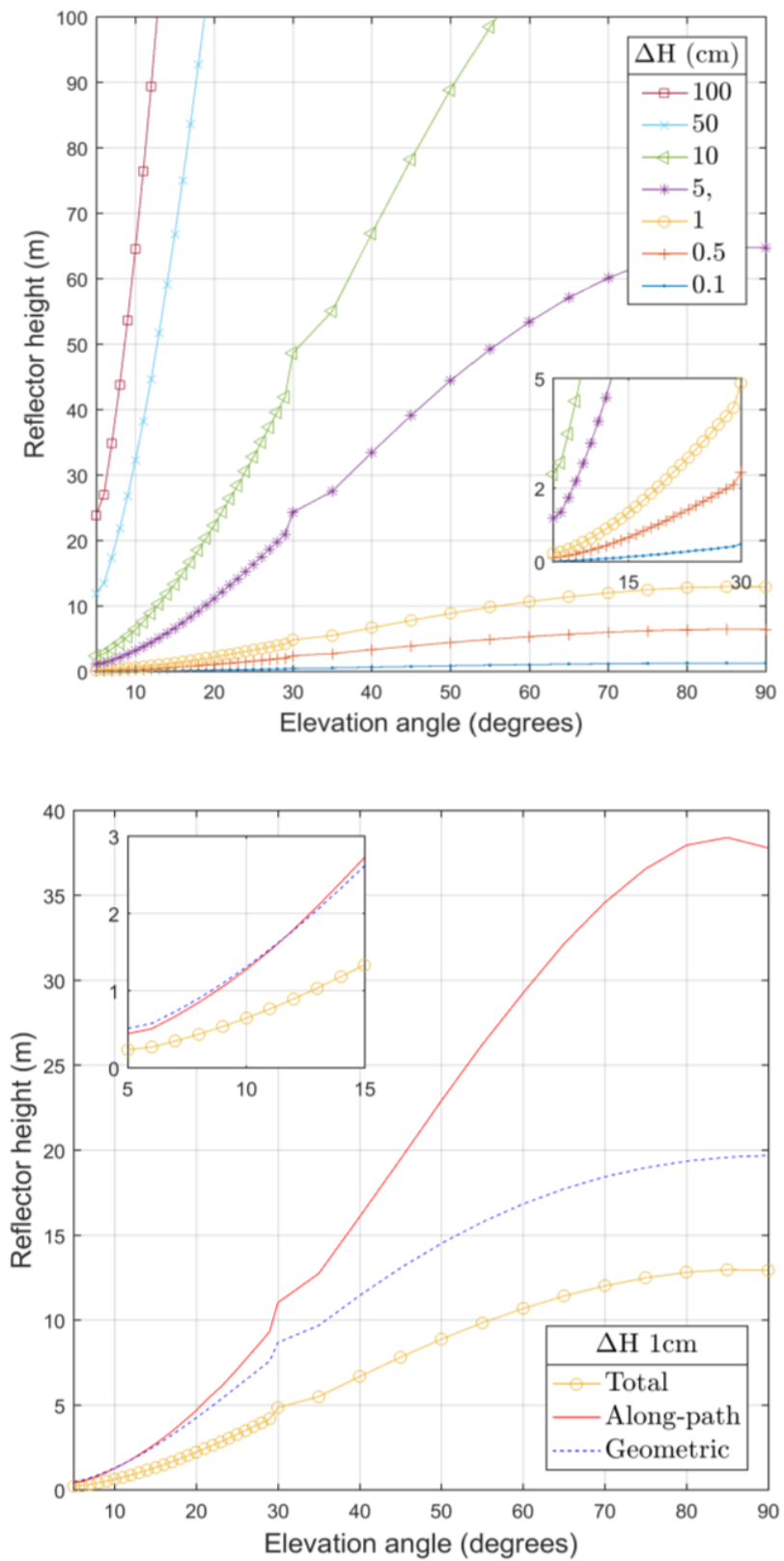

Fig. 5: Closed-form atmospheric altimetry correction as contour lines over domain of satellite elevation angle and reflector height; top: for multiple contour lines of total correction; bottom: for $1-\mathrm{cm}$ contour line of total correction and its components.

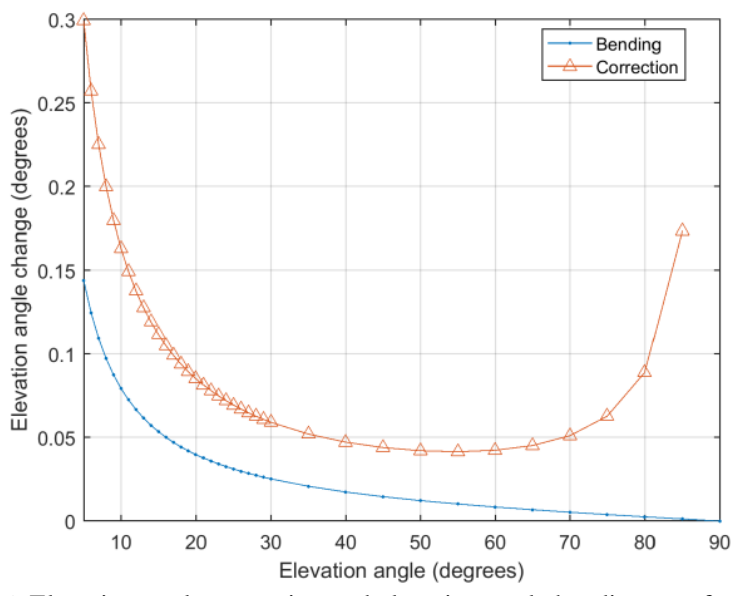

Fig. 6: Elevation angle correction and elevation angle bending as a function of satellite elevation angle. 


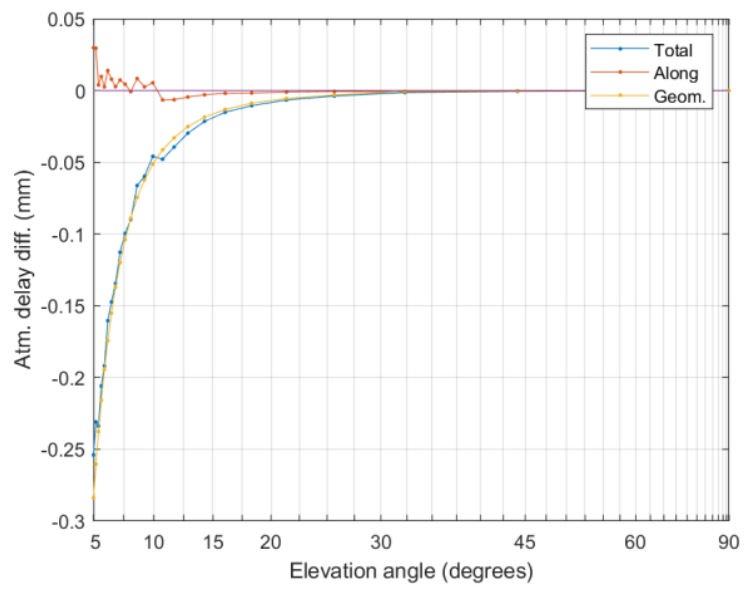

Figure S1: Discrepancy in interferometric atmospheric delay between rectilinear and rigorous raytracing as a function of satellite elevation angle (for a fixed 10-m reflector height).

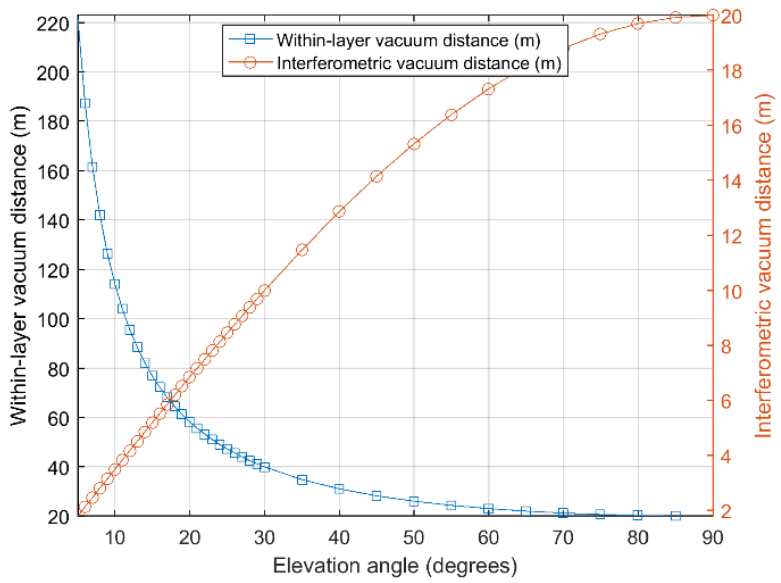

Fig. S2: Layer slant distance (left axis) and interferometric vacuum distance (right axis), both as a function of satellite elevation angle (for a fixed 10-m reflector height).

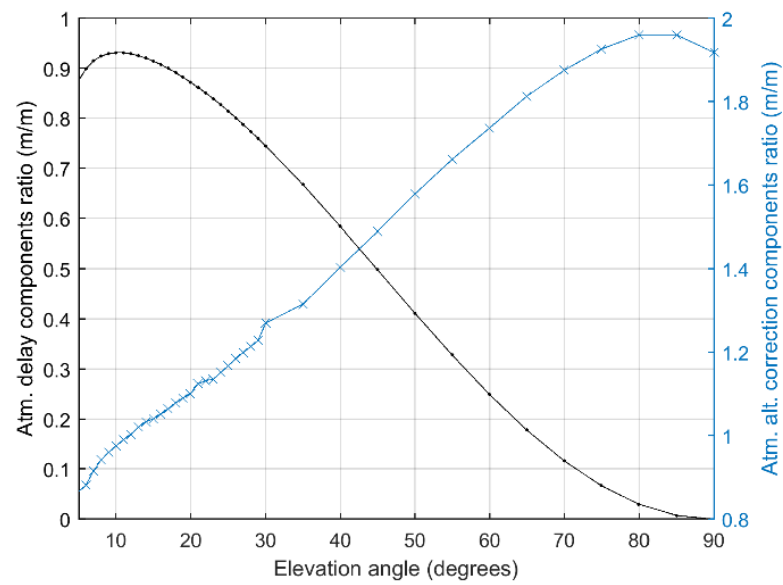

Fig. S3: Ratio of closed-form atmospheric delay components (black dots) and ratio of closed-form altimetry correction components (blue crosses) - both in the order geometric over along-path - as a function of satellite elevation angle. 